\title{
Power conversion factor in solar flares
}

\author{
NING ZongJun ${ }^{1,2}$ \\ ${ }^{1}$ Key Laboratory of Dark Matter and Space Astronomy, Chinese Academy of Sciences, Nanjing 210008, China; \\ ${ }^{2}$ Purple Mountain Observatory, Chinese Academy of Sciences, Nanjing 210008, China
}

Received September 16, 2011; accepted January 5, 2012

\begin{abstract}
With RHESSI data from five solar flares taken from beginning to end, we investigate the power conversion factor $\mu$ defined as the ratio of the time derivative of total thermal energy $\left(E_{\text {RHESSI }}+E_{\text {rad }}+E_{\text {cond }}\right)$ and the kinetic power $\left(P_{\text {RHESSI }}\right)$ of nonthermal electrons. Here, $E_{\text {RHESSI }}$ is the computed energy contained in thermal plasmas traced by RHESSI SXRs. Other two contributions $\left(E_{\text {rad }}\right.$ and $\left.E_{\text {cond }}\right)$ to the total energy are the energies lost through radiation and conduction, both of which can be derived from the observational data. If both are not considered, $\mu$ is only positive before the SXR maximum. However, we find that for each flare studied $\mu$ is positive over the whole duration of the soalr flare after taking into account both radiation and conduction. Mean values for $\mu$ range from $11.7 \%$ to $34.6 \%$ for these five events, indicating roughly that about this fraction of the known energy in nonthermal electrons is efficiently transformed into thermal energy from start to end. This fraction is traced by RHESSI SXR observations; the rest is lost. The bulk of the nonthermal energy could heat the plasma low in the atmosphere to drive mass flows (i.e. chromospheric evaporation).
\end{abstract}

solar flare, X-ray, thermal energy, nonthermal power

Citation: Ning Z J. Power conversion factor in solar flares. Chin Sci Bull, 2012, 57: 1397-1404, doi: 10.1007/s11434-012-5058-6

It is well known among solar flare researchers of the existence of hot (temperature up to tens of MK), dense plasma confined within coronal loops or series of loops. These plasma provide strong emissions of soft X-rays (SXRs) which are recorded by the Reuven Ramaty High Energy Solar Spectroscopic Imager (RHESSI). There is strong evidence for the coronal plasma heating from nonthermal electrons accelerated by current sheets within solar flares. Based on the standard model, a major contribution of the energy is first released from the energetic, nonthermal electrons and possibly plasma ions. These particles, guided by magnetic field lines, can escape into interplanetary space, but can also be precipitated into the lower corona or upper chromosphere where by Coulomb collision kinetic energy is exchanged with the denser medium, thereby rapidly heating the local plasma. The resulting overpressure drives an upward mass flow, which the SXRs actually trace out, to fill the flaring loops with hot plasma. An intimate relationship between thermal and nonthermal time profiles exists as often both profiles closely resemble each

email: ningzongjun@pmo.ac.cn other. Observationally, the derivatives of the SXR light curve correspond well with the hard X-ray (HXR) and microwave burst profiles, dubbed the Neupert effect [1-7], suggesting that the flaring thermal energy traced by the SXRs is transformed from kinetic energy of nonthermal electrons producing HXR and microwave bursts. In the absence of cooling (radiation and conduction) or long timescales [8], the Neupert effect suggests that the thermal energy reaches its maximum almost at the same time as the end of the nonthermal energy input. In actual flare scenarios, the radiative and conductive cooling could start after flare onset, resulting in flare temperatures, emission measures, and thermal energies attaching their maximum levels before the end of nonthermal energy input. Typically, flaring temperatures reach maximum levels a few minutes before the other two $[9,10]$.

One issue of interest is to compare the thermal energy of flaring plasma with the total kinetic energy of nonthermal electrons that are precipitated in solar flares. In the flare framework, the nonthermal power $P_{\text {nth }}$ (expressed here in the units of $\mathrm{erg} \mathrm{s}^{-1}$ ) represents the instantaneous energy deposition rate by the electron beam precipitating out into the chro- 
mosphere, but the thermal energy $E_{\text {th }}$ (in the units of erg) is proportional to the cumulative energy deposited; that is, $E_{\mathrm{th}}$ is the time integral of $P_{\text {nth }}$. Thus, the Neupert relationship can be expressed as

$$
\frac{\mathrm{d} E_{\mathrm{th}}(t)}{\mathrm{d} t}=\mu(t) P_{\mathrm{nth}(t)},
$$

where the coefficient $\mu(t)$ is the power conversion rate. Observationally, the thermal $\left(E_{\mathrm{th}}\right)$ and nonthermal time-integrated $\left.\mu P_{\text {nth }}\right)$ are usually derived from the soft and HXR emissions respectively [11-18]. However, these two emissions are cospatial and overlap in photon energies in the range from 10 to $25 \mathrm{keV}$. The distinction between the two forms of energy requires a high spectral resolution in the critical range. With its full disk solar imaging capability, wide energy coverage, high temporal resolution and high sensitivity, RHESSI [1921] provides unprecedented capabilities to separate nonthermal components from thermal continuum with much higher precision than before. Using the RHESSI data, Saint-Hilaire and Benz [13] studied thermal and nonthermal energies in 14 flares, and obtained a nonthermal/thermal ratio ranging from 0.7 to 6.3 . Within a flare, this value increases as time progresses, as expected if thermal energy decreases due to radiative and conductive cooling. Using a constant accumulation time, Ning and Cao [16] studied the relation between thermal and nonthermal energies from flare onset. They concluded that about $2 \%-20 \%$ of nonthermal energy traced by HXRs is transformed into the thermal energy to produce the SXRs.

Theoretically, the Neupert effect suggests that flare thermal energy $\left(E_{\mathrm{th}}\right)$ should increase linearly with time during HXR emissions (or nonthermal energy input). Observationally, due to the radiation and conduction cooling, the thermal energy derived from the data usually starts to decrease before HXR emissions end. For example, Ning and Cao [16] found that the rate of change of the computed energy $E_{\mathrm{RHESSI}}$ contained in thermal plasmas traced by RHESSI SXRs, $\mathrm{d} E_{\mathrm{RHESSI}} / \mathrm{d} t$, becomes negative after $E_{\text {RHESSI }}$ peaks while HXR emissions were still being observed. Taking cooling into account, $E_{\text {th }}$ should be total of $E_{\text {RHESSI }}$ traced by the SXRs and the energy by the radiation and conduction, i.e. $E_{\text {rad }}$ and $E_{\text {cond }}$, which can be estimated from the RHESSI observations. Thus, from RHESSI observations, eq. (1) has the following from:

$$
\frac{\mathrm{d}\left(E_{\mathrm{RHESSI}}(t)+E_{\text {cond }}(t)+E_{\mathrm{rad}}(t)\right)}{\mathrm{d} t}=\mu(t) P_{\mathrm{RHESSI}}(t) .
$$

In this paper, we examines five RHESSI flares to obtain both radiation and conduction energy rates with the aim to analyze power conversion factor between the nonthermal power (in the following $P_{\text {RHESSI }}$ is used instead of $P_{\text {nth }}$ ) and the total thermal energy rates after flare onset.

\section{Observations and measurements}

The events we studied included an X-class event on 2005 September 13, and four M-class events on 2003 November
13, 2004 November 4, 2005 August 3 and 25 respectively. Table 1 lists their information from GOES and RHESSI observations. RHESSI observes in a broad range from X-rays to $\gamma$-rays. Its high spectral resolution $(1 \mathrm{keV}$ in the $\mathrm{X}$-ray range) and coverage of the low-energy range (down to 3 $\mathrm{keV}$ ) enable us to derive thermal and nonthermal quantities from specta, and to study flare temperature, emission measure, spectral index, low-energy cutoff ( $\left.E_{\text {cutoff }}\right)$, thermal and nonthermal energies after flare onset. The RHESSI standard code OSPEX is used to perform spectral fits. Figure 1 shows examples of RHESSI spectral fittings over two time intervals for the 2005 September 13 flare. We use a photon-spectrum model comprising a thermal continuum and a thick-target bremsstrahlung component (no line emission) in the energy range between 6 and $80 \mathrm{keV}$. Before a spectral fitting, we generated RHESSI count spectrograms for each flare in the uninterrupted sunlight time interval with time binning of 10 $\mathrm{s}$, and energy binning of $1 \mathrm{keV}$ over energies 3 to $20 \mathrm{keV}$, and $2 \mathrm{keV}$ from 20 to $300 \mathrm{keV}$. We use only the front segments detectors $3-8$ excluding 7 . This spectral fitting is same as for other four events.

The 2005 September 13 flare is a typical event that occurred on the decay phase of the last 23th solar cycle. According to GOES observations, it is an X1.7 Class starting at 23:15 UT with a duration of 15 minutes. Several groups have analyzed this event and associated phenomena, such as the related radio bursts [22], CME [23,24], filament [25], and magnetic configuration [26]. There are two subpeaks at 23:18:30 UT and 23:19:50 UT. Figure 2 shows the SOHO/MDI magnetogram at 23:23:03 UT with RHESSI contours at 23:18:00 UT (a) and 23:19:30 UT (b) respectively. The levels are set at $70 \%, 80 \%$, and $90 \%$ for $6-9 \mathrm{keV}$ (black) and 40\%, 60\%, and $80 \%$ for $50-100 \mathrm{keV}$ (white). This event displays a thermal looptop source at 6-9 keV localized between two footpoint sources at 50-100 keV. Such morphology is typical of solar flares seen in X-rays. It is interesting to note that the flaring loop seen with X-rays is almost parallel to the magnetic neutral line (white dotted line). This fact is different from the general picture for solar flares which put their HXR footpoints on both sides of the neutral line. A detailed description of the magnetic field and flare ribbon can be found in [26].

Figure 3(a)-(i) gives the temporal evolution of the X-ray emissions and the derived parameters for the 2005 September 13 flare that we used for this paper, including the light curves of RHESSI X-ray emissions at five energy bands of $6-12 \mathrm{keV}, 12-25 \mathrm{keV}, 25-50 \mathrm{keV}, 50-100 \mathrm{keV}$, and $100-$ $300 \mathrm{keV}$, and GOES SXR flux at $1-8 \AA$ (dotted) in panel (a); the temperature from GOES (dotted) and RHESSI (triangle) in panel (b); the emission measure from GOES (dotted) and RHESSI (diamond) in panel (c); the radiative $\left(P_{\text {rad }}\right.$, dot-dashed) and conductive ( $P_{\text {cond }}$, dashed) cooling rates and their total $\left(P_{\text {rad }}+P_{\text {cond }}\right.$, plus $)$ derived from RHESSI, the total cooling rate detected from GOES data $\left(P_{\mathrm{GOES}}\right.$, solid $)$ in panel (d); the observational energies detected from GOES ( $E_{\mathrm{GOES}}$, dotted), from RHESSI ( $E_{\text {RHESSI}}$, triangle), and the energy 

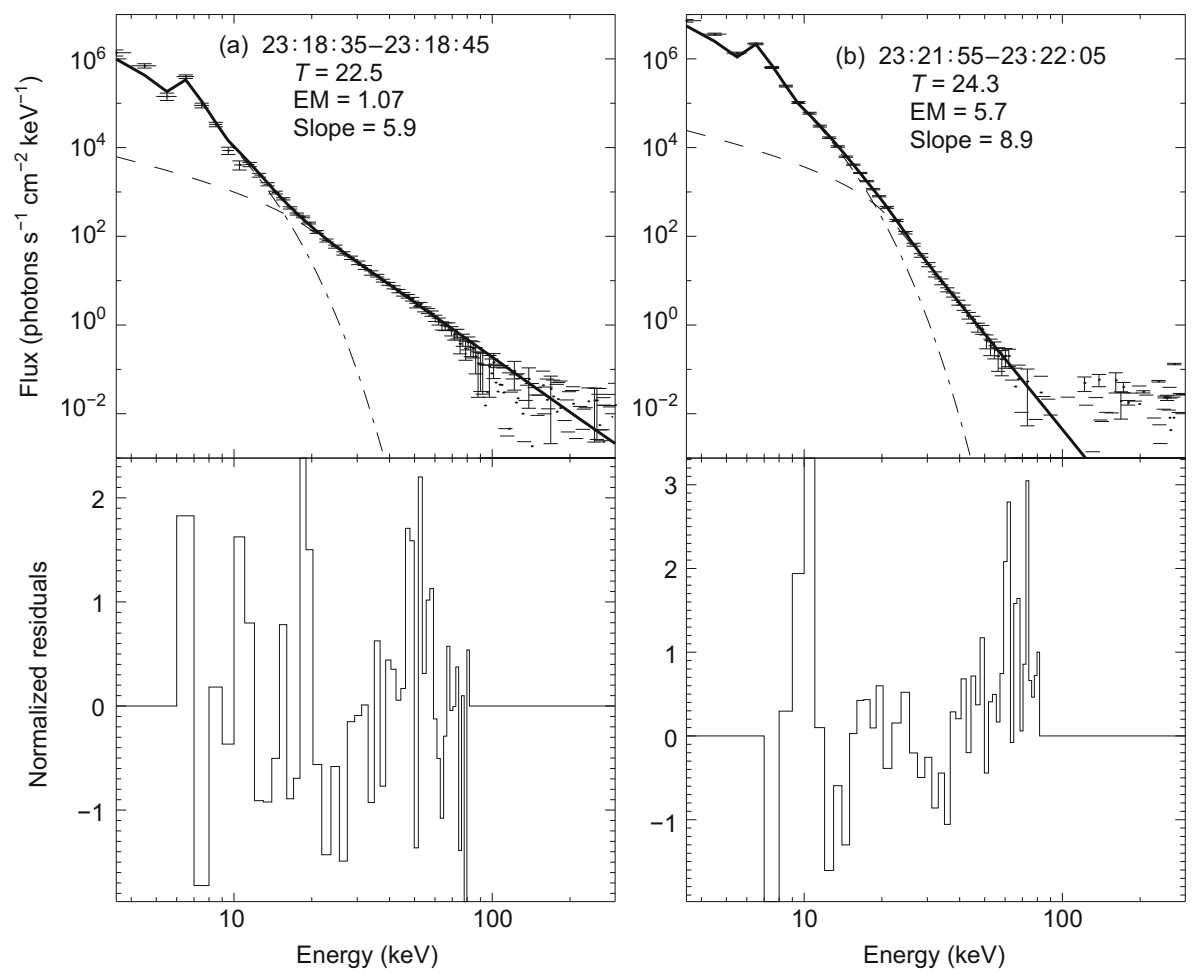

Figure 1 Top panels: RHESSI spectra at two discrete time intervals for the 2005 September 13 flare. The points with error bars represent the observational spectral data. The lines represent model spectral fits, the dot-dashed line representing the thermal component, the dashed line the nonthermal thick-target bremsstrahlung, and the thick solid line is the sum of the two. The legend gives fitting parameters for temperature ( $T$ in units of MK), emission measure (EM in units of $10^{49} \mathrm{~cm}^{-3}$ ), and power-law slope. Bottom panels: Normalized residuals in the energy range required for spectral fitting.
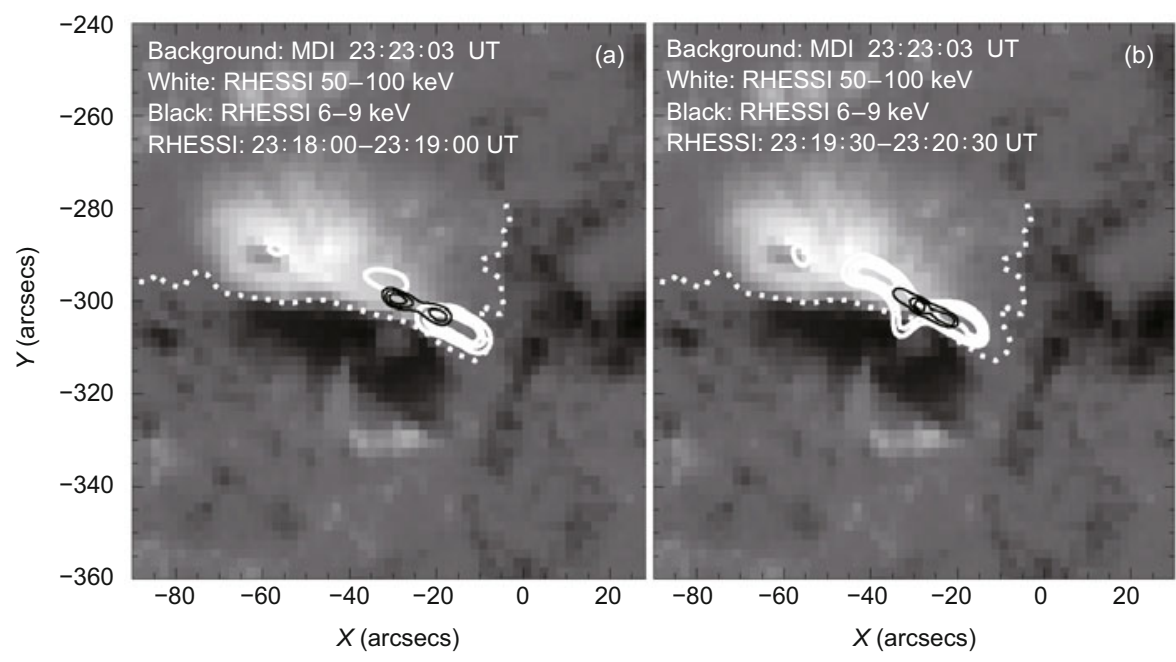

Figure 2 SOHO/MDI magnetogram at 23:23:03 UT with RHESSI X-ray contours at 23:19:30 UT for the 2005 September 13 solar flare. The white dotted line represents the magnetic neutral line. The contour levels are set at $70 \%, 80 \%$ and $90 \%$ for $6-9 \mathrm{keV}$ (black), and $40 \%, 60 \%$ and $80 \%$ for $50-100 \mathrm{keV}$ (white).

lost through radiation $\left(E_{\mathrm{rad}}\right.$, dot-dashed) and conduction ( $E_{\text {cond }}$, dashed) detected from RHESSI, the total energy lost ( $E_{\text {lose }}$, solid) deduced from GOES in panel (e); the ratios of the energy loss to the computed energy, i.e., $\frac{E_{\mathrm{rad}}+E_{\mathrm{cond}}}{E_{\mathrm{RHESSI}}}$ (solid line) detected from RHESSI, and $\frac{E_{\mathrm{loss}}}{E_{\mathrm{GOES}}}$ (dot line) deduced from GOES in panel (f); the electron power-law index (thin line) and low energy cutoff ( $E_{\text {cutoff }}$, star) in panel $(\mathrm{g})$; the nonthermal energy power $\left(P_{\text {RHESSI}}\right.$, plus) detected from RHESSI, the derivative of RHESSI thermal energy ( $\mathrm{d} E_{\mathrm{RHESSI}} / \mathrm{d} t$, star), of total thermal energies from RHESSI $\left(\mathrm{d}\left(E_{\text {RHESSI }}+E_{\text {rad }}+E_{\text {cond }}\right) / \mathrm{d} t\right.$, diamond $)$, and from GOES $\left(\mathrm{d}\left(E_{\mathrm{GOES}}+E_{\text {lose }}\right) / \mathrm{d} t\right.$, solid $)$ in panel $(\mathrm{h})$; the ratios of $\frac{\mathrm{d} E_{\text {RHESSI }} / \mathrm{d} t}{P_{\text {RHESSI }}}$ (star) and $\frac{\mathrm{d}\left(E_{\text {RHESSI }}+E_{\text {rad }}+E_{\text {cond }}\right) / \mathrm{d} t}{P_{\text {RHESSI }}}$ (diamond) in panel (i). As commonly observed, using GOES data for detecting the thermal parameters yields a high EM and a lower $T$ than RHESSI $[27,28]$. 


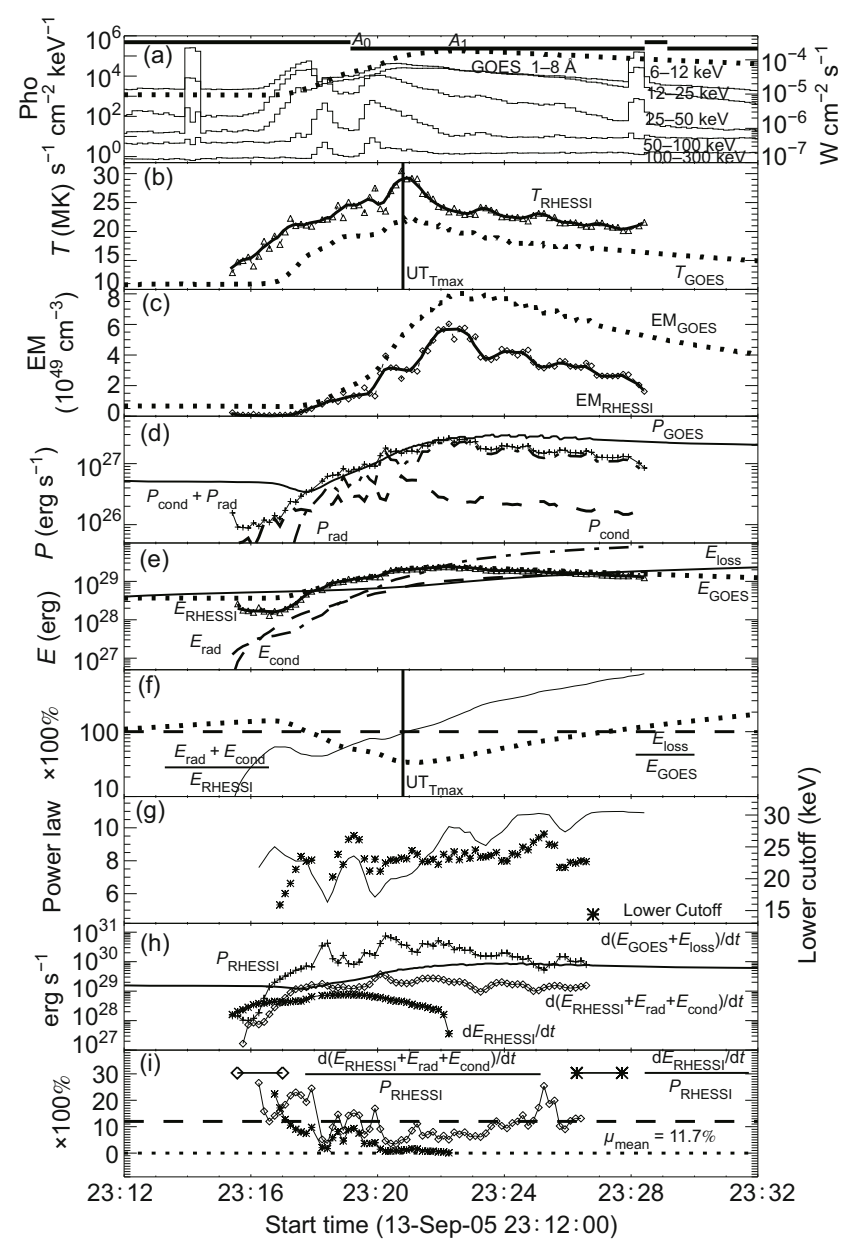

Figure 3 Time evolution of all parameters for the 2005 September 13 solar flare. $T_{\text {RHESSI }}, \mathrm{EM}_{\mathrm{RHESSI}}, P_{\text {rad }}, P_{\text {cond }}, E_{\mathrm{RHESSI}}, E_{\mathrm{rad}}, E_{\text {cond }}$, power index, low-energy cutoff ( $\left.E_{\text {cutoff }}\right)$, and $P_{\text {RHESSI }}$ are derived from RHESSI data; $T_{\mathrm{GOES}}, \mathrm{EM}_{\mathrm{GOES}}, P_{\mathrm{GOES}}, E_{\mathrm{GOES}}$ and $E_{\text {lose }}$ are derived from GOES data. See definitions of terms in Section 2 of the text. The solid line on panel (a) shows the attenuator states $\left(\mathrm{A}_{0}\right.$ or $\left.\mathrm{A}_{1}\right)$. The dot-dot-dot-dashed lines in panels $(\mathrm{b})$ and (f) mark the positions of maximum temperature. The dotted line in panel (i) represents the zero level, and the dashed line shows the mean value of parameter $\mu$.

As noted earlier, the temperature, emission measure, spectral index and low-energy cutoff are computed for each interval from OSPEX code. These are treated as free parameters used in spectral fitting. Figure 3(b) shows that the 2005 September 13 flare reached a maximum temperature ( $\left.T_{\max }\right)$ of $30.5 \mathrm{MK}$; this event follows a soft-hard-soft (SHS) spectral pattern in the rising-peak-decay phases of each subpeak as shown in panel $(\mathrm{g})$. The low-energy cutoff $\left(E_{\text {cutoff }}\right)$ is around $23 \mathrm{keV}$. The thick solid lines overplotted in Figure 3(b), (c), (e), (h) represent the smoothed profiles using a time window of $30 \mathrm{~s}$. The thermal energy $\left(E_{\text {RHESSI }}\right)$ is detected from RHESSI data using an usual way [7,14,29,30], $E_{\text {RHESSI }}(t)=3 n_{e}(t) V(t) k T(t)=3 k T(t) \sqrt{E M(t) \times V(t) \times f}$, where $k$ is the Boltzmann constant, $n_{e}(t)$ the plasma density, and $V(t), \operatorname{EM}(t)\left(=n_{e}^{2}(t) V(t)\right)$, and $T(t)$ are the source volume, emission measure, and temperature of the thermal plasma respectively. Here we assume that the filling factor of the hot plasma is of order unity; i.e. $f=1$. The flare volume is approximately estimated from the source area in the RHESSI CLEAN images [7,14,29]; i.e., $V=A^{3 / 2}$, where $A$ is the source area inside the $50 \%$ contour of the $6-9 \mathrm{keV}$ image. According to this method, the flaring volume does not change much during flare development. For example, the estimated source volume is about $10^{28}$ for these five flares studied in this paper. This value of flare volume is also used to detect the GOES thermal energy of $E_{\mathrm{GOES}}$ (dotted line) in panel e. The nonthermal energy power of $P_{\text {RHESSI }}$ is computed for each time interval by integrating the electron power-law spectrum above the low-energy cutoff [16,26,29].

The radiative and conduction colling rates, $\left(P_{\mathrm{rad}}=\mathrm{d} E_{\mathrm{rad}} / \mathrm{d} t\right.$ and $\left.P_{\text {cond }}=\mathrm{d} E_{\text {cond }} / \mathrm{d} t\right)$, are calculated from formulas: $P_{\text {rad }}=$ $6 \times 10^{-22} \times E M / \sqrt{T} \mathrm{erg} \mathrm{s}^{-1}$ and $P_{\text {cond }}=4 \times 10^{-6} \times \sqrt{T^{7}} / L$ erg s${ }^{-1}[8,11,28,31-34]$, where $L \sim 10^{-6} \mathrm{~cm}^{-1}$ is the temperature scale length, assumed constant within the solar atmosphere [6]. Consistent with previous findings, the 2005 September 13 flare shows that the conductive cooling dominates in the impulsive phase, and the radiative cooling dominates in the later phase $[8,9,34,35]$. After obtaining $P_{\text {rad }}$ and $P_{\text {cond }}$, the Neupert effect (or energy balance) requirement can be simply addressed by comparing the net energy input rate $P_{\text {RHESSI }}-\left(P_{\text {rad }}+P_{\text {cond }}\right)$ with the change in the energy content $\mathrm{d} E_{\mathrm{RHESSI}} / \mathrm{d} t$. In other words, eq. (2) becomes

$$
\frac{\mathrm{d} E_{\mathrm{RHESSI}}(t)}{\mathrm{d} t}=\mu(t)\left(P_{\mathrm{RHESSI}}(t)-P_{\mathrm{rad}}(t)-P_{\text {cond }}(t)\right) .
$$

In this case, $\mu(t)$ can not be derived if $\mathrm{d} E_{\mathrm{RHESSI}} / \mathrm{d} t$ becomes negative after $E_{\text {RHESSI }}$ has peaked. However, HXR emissions are still observed. This motivates our use of eq. (2) to derive $\mu(t)$.

After integration of the cooling rates in each interval, the energy lost through radiation $\left(E_{\mathrm{rad}}\right)$ and conduction $\left(E_{\text {cond }}\right)$ are calculated for each interval as follows: $E_{\text {rad }}=$ $\int_{t 0}^{t} P_{\mathrm{rad}}(i) \mathrm{d} i \times \delta t$ and $E_{\text {cond }}=\int_{t 0}^{t} P_{\text {cond }}(i) \mathrm{d} i \times \delta t$. Here $t_{0}$ is the start time of the spectral fitting, and $\delta t$ is the same as the time binning $(10 \mathrm{~s})$ for the RHESSI light curves in this paper. Time evolutions for $E_{\text {rad }}$ and $E_{\text {cond }}$ are shown in panel (e). At flare onset, the input energy is remarkably greater than the energy lose, resulting in an increase in flare temperature. After a period, the temperature peaks as radiation and conduction energies become comparable with the input energy. Figure 3(e) shows the radiation energy $\left(E_{\mathrm{rad}}\right)$ is nearly comparable with the observational thermal energy $\left(E_{\text {RHESSI }}\right)$ in the later phase of the flare. The temperature decreases monotonously after peaking at time $\mathrm{UT}_{T_{\max }}$. Figure 3(f) showing that $\mathrm{UT}_{T_{\max }}$ is almost the same as the time when $\frac{E_{\mathrm{rad}}+E_{\mathrm{cond}}}{E_{\mathrm{RHESSI}}}=1$, indicating that radiation and conduction are the main cooling mechanisms in this flare.

\section{Thermal and nonthermal energies}

Figure 3(e) shows for the 2005 September 13 event the time evolution of flare energies, including $E_{\text {RHESSI }}$ (the observa- 
tional thermal energy), $E_{\text {rad }}$ (radiation), $E_{\text {cond }}$ (conduction), and $P_{\text {RHESSI }}$ (nonthermal energy input). $E_{\text {rad }}$ and $E_{\text {cond }}$ almost increase monotonously with time. Thus, the flare attains an energy balance between $E_{\text {rad }}+E_{\text {cond }}$ and $E_{\text {RHESSI }}$, at which time the flare temperature peaks. After reaching peak temperature $\left(U T_{T_{\max }}\right)$, the energy of $E_{\mathrm{rad}}+E_{\text {cond }}$ is greater than the computed energy of $E_{\text {RHESSI }}$. This can be seen in panel (f) which plots the ratio of energy loss $\left(E_{\text {rad }}+E_{\text {cond }}\right)$ to deduced energy ( $\left.E_{\text {RHESSI }}\right)$. This ratio shows a simple trend, in particular after $U T_{T_{\max }}$. At flare end (which means the final time that any spectral fitting can be done well), $E_{\text {rad }}+E_{\text {cond }}$ is about $700 \%$ times $E_{\text {RHESSI}}$, indicating that the bulk of flare thermal energy is lost. In other words, the computed energy is only about $12.5 \%$ of the total thermal energy at flare end.

To derive the power conversion factor $(\mu)$ from the nonthermal to thermal energies, $E_{\text {rad }}$ and $E_{\text {cond }}$ have to be considered. The total thermal energy of $E_{\mathrm{RHESSI}}+E_{\mathrm{rad}}+E_{\text {cond }}$ is used to calculate the value of $\mu$. In this case, $\mu=$ $\frac{\left(E_{\text {RHESSI }}+E_{\text {rad }}+E_{\text {cond }}\right) / \mathrm{d} t}{P_{\text {RHESSI}}}$, as the line with diamonds in Figure 3(i). The line with star shows the time evolution of the ratio of $\frac{E_{\text {RHESSI }} / \mathrm{d} t}{P_{\text {RHESSI }}}$. Consistent with previous studies [16], it becomes negative after the maximum of $E_{\text {RHESSI }}$. After considering the radiation and conduction, the value of $\mu(t)$ is positive during the flare lifetime. $\mu(t)$ has a mean value of $11.7 \%$ as shown

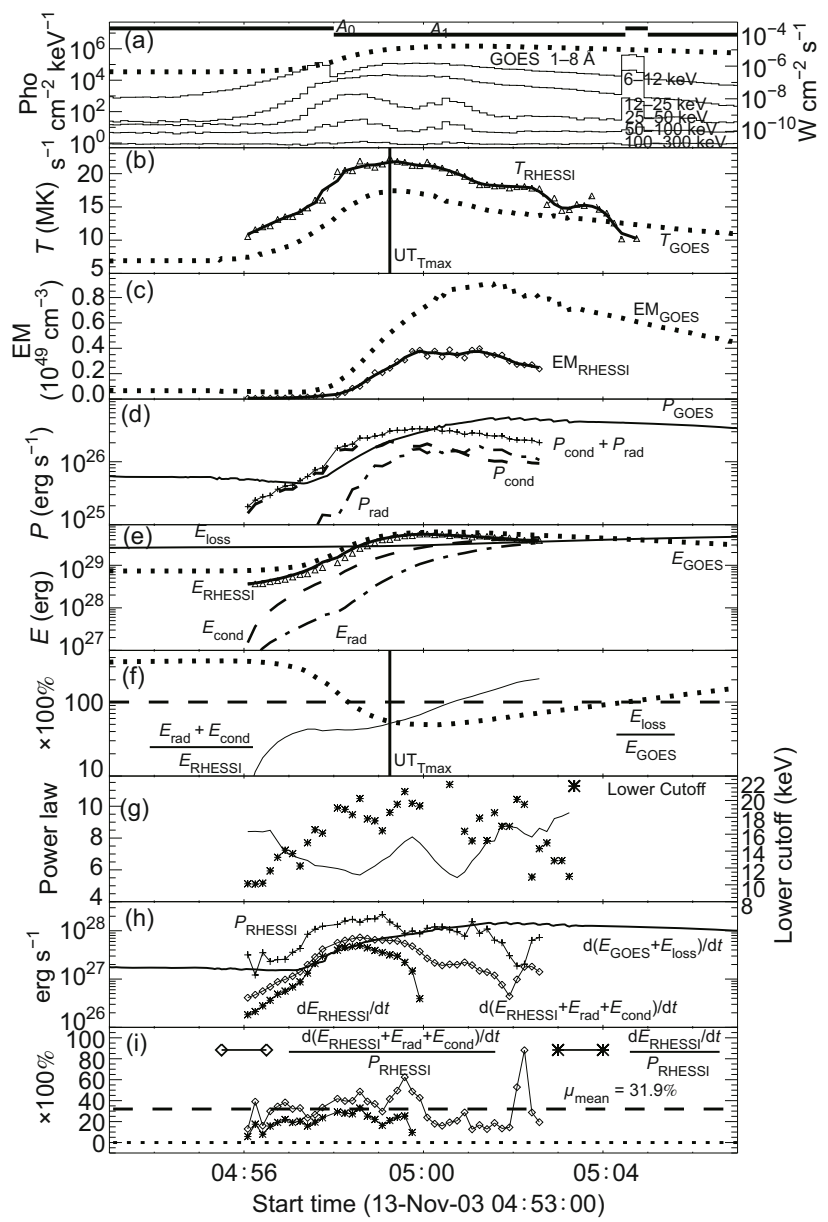

Figure 4 Same as Figure 3, but for the 2003 November 13 solar flare. with the dashed line, although $\mu(t)$ displays a small variation with the time. It is an expectation from the Neupert effect that the energy of the flare thermal plasma is transformed from the kinetic energy of nonthermal electrons from the flare start to end.

Using the same method, Figures 4-7 show the time evolution of the flare parameters and the power conversion rate of $\mu(t)$ computed for the 2003 November 13, 2004 November 4, 2005 August 3 and 25 flares. To compare with RHESSI data, the corresponding parameters derived from GOES data are also plotted in these figures. Similar to the 2005 September 13 flare, these four events display the SHS spectral pattern during the rising-peak-decay phases of the subpeaks. Their maximum temperatures, listed in Table 1, are around $23 \mathrm{MK}$. The energy loss $E_{\text {rad }}+E_{\text {cond }}$ increases with time, and becomes comparable with the computed energy of $E_{\text {RHESSI }}$ around $U T_{T_{\max }}$ for each event. At each flare end, the ratio $\frac{E_{\mathrm{rad}}+E_{\mathrm{cond}}}{E_{\mathrm{RHESSI}}}$ ranges from $200 \%$ to $800 \%$ for these four events. Thus, only a small fraction (11\%-33\%) of the thermal energy is obtained from RHESSI observations at SXRs, the bulk must be lost to radiation and conduction. In contrast to single temperature peaks seen in other four events, the 2005 August 3 event shows a pair of temperature peaks, the second being greater than the first. During the period between these peaks,

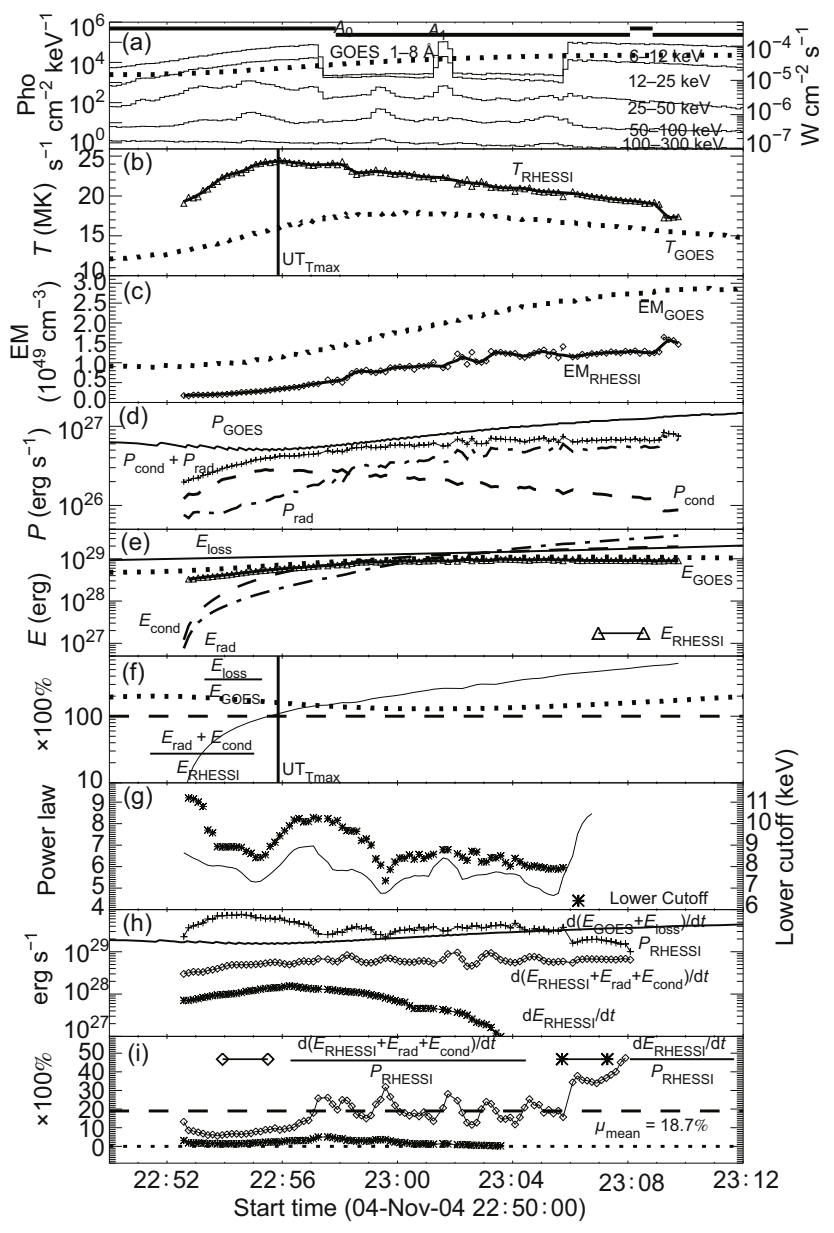

Figure 5 Same as Figure 3, but for the 2004 November 4 solar flare. 


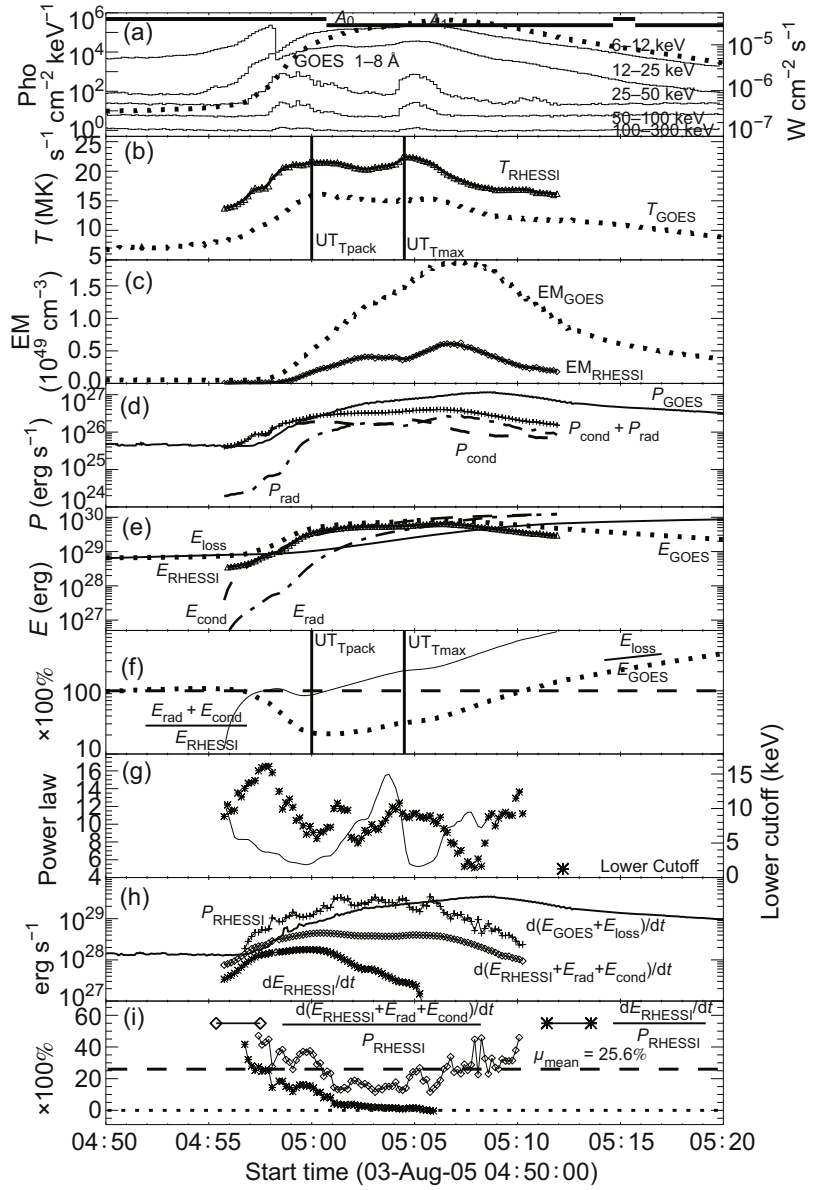

Figure 6 Same as Figure 3, but for the 2005 August 3 solar flare.

temperatures decrease. Figure 6(f) shows that the balance between $E_{\text {rad }}+E_{\text {cond }}$ and $E_{\text {RHESSI }}$ almost at the first temperature peak of $U T_{T_{\text {peak }}}$.

Similar to the 2005 September 13 flare, these four events show a positive $\mu(t)$ from start to finish. The mean values of $\mu(t)$ are $31.9 \%, 18.7 \%, 25.6 \%$ and $34.5 \%$, as listed in Table 1. The average $\mu(t)$ of these five events is $\mu(t)=24.5 \%$. Figure 8 plots $P_{\text {RHESSI }}$ against d $\left(E_{\text {RHESSI }}+E_{\text {rad }}+E_{\text {cond }}\right) / \mathrm{d} t$ for the five flares studied. Consistent with the flare model, the nonthermal energy power $P_{\text {RHESSI }}$ is greater than the time rate of change of total thermal energy d $\left(E_{\text {RHESSI }}+E_{\text {rad }}+E_{\text {cond }}\right) / \mathrm{d} t$ in all flares. The solid line represents the linear fit for a log-log plot of the data. A high correlation of 0.86 is found, and the positive dependence suggests that these five events follow the Neupert effect.

Table 1 List of flares

\begin{tabular}{cccccccc}
\hline Date & Start-end & Class & NOAA Spectra & $\begin{array}{c}T_{m} \\
(\mathrm{MK})\end{array}$ & $\begin{array}{c}E_{c} \\
(\mathrm{keV})\end{array}$ & $\mu$ \\
\hline 2003-11-13 & $04: 54-05: 06$ & M1.6 & 10501 & SHS & 22.3 & 19.4 & $31.9 \%$ \\
$2004-11-04$ & $22: 53-23: 26$ & M5.4 & 10696 & SHS & 24.5 & 10.2 & $18.7 \%$ \\
$2005-08-03$ & $04: 54-05: 11$ & M3.2 & 10794 & SHS & 22.4 & 8.6 & $25.6 \%$ \\
2005-08-25 & $04: 31-04: 45$ & M6.4 & 10803 & SHS & 24.4 & 8.8 & $34.5 \%$ \\
$2005-09-13$ & $23: 15-23: 30$ & X1.7 & 10808 & SHS & 30.5 & 20.4 & $11.7 \%$ \\
\hline
\end{tabular}

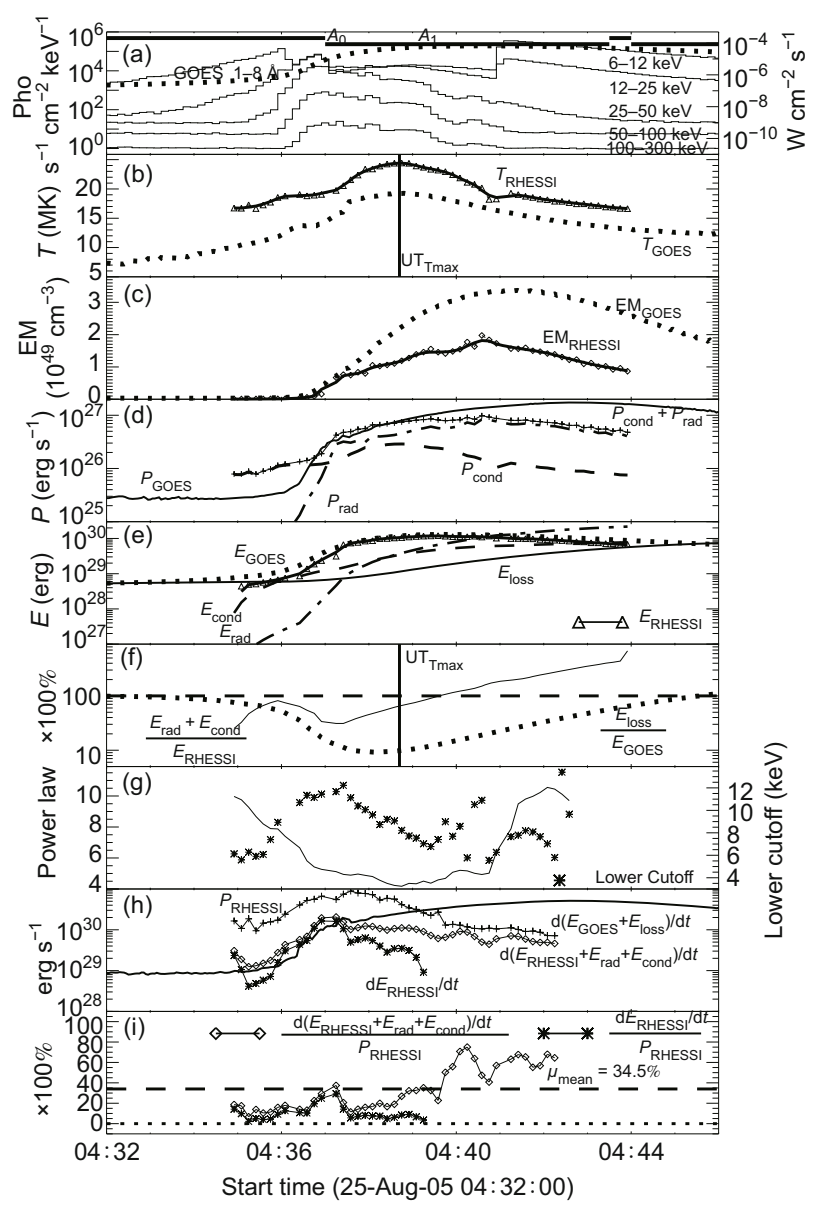

Figure 7 Same as Figure 3, but for the 2005 August 25 solar flare.

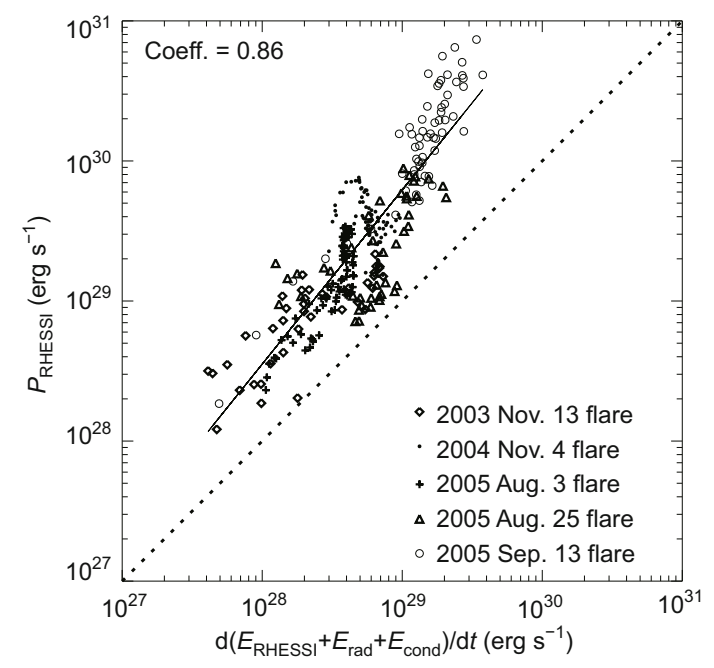

Figure 8 Correlation plots of the time rate of change of the total thermal energy $\left(E_{\mathrm{RHESSI}}+E_{\mathrm{rad}}+E_{\text {cond }}\right)$ dependence on the nonthermal energy input of $P_{\text {RHESSI }}$ in the log-log frame for five solar flares. The solid line represents a linear fit of all data.

\section{Conclusions and discussion}

Previous studies have discovered that radiative and conductive cooling play an important role in solar flaring. For exam- 
ple, these cooling processes can cause thermal delays, as an analysis of RHESSI data in the paper by Aschwanden [34] has shown. Using the RHESSI data, the radiation and conduction are measured after flare onset in five events. As a usual, the cooling is too weak to be worth considering initially, resulting into a positive dependence found between the time rate of change of derived thermal energy $\left(\mathrm{d} E_{\mathrm{RHESSI}} / \mathrm{d} t\right)$ and nonthermal power $\left(P_{\text {RHESSI }}\right)$. Cooling then gradually increases with time, resulting in their negative dependence in later phases of flaring [16].

For this paper, power conversion from kinetic energy of nonthermal electrons to total thermal energy has been measured in five solar flares. Distinct from previous studies $[13,14,16]$ in which only the thermal energy ( $\left.E_{\text {RHESSI }}\right)$ directly deduced from the RHESSI SXRs is used, the energy loss by radiative and conductive cooling are considered here. According to our method, the balance between the energy loss and observational thermal energy is around temperature peaking, indicating that the assessment of radiation and conduction are reasonable in this paper. Meanwhile, the total thermal energy $\left(E_{\text {RHESSI }}+E_{\text {rad }}+E_{\text {cond }}\right)$ and the nonthermal energy input $\left(P_{\text {RHESSI }}\right)$ are determined in each flare from start to end. In this case, the ratio $\mathrm{d}\left(E_{\mathrm{RHESSI}}+E_{\text {rad }}+E_{\text {cond }}\right) / \mathrm{d} t$ to $P_{\text {RHESSI }}$ is always positive during solar flaring. In other words, a positive dependence of $\mathrm{d}\left(E_{\mathrm{RHESSI}}+E_{\mathrm{rad}}+E_{\text {cond }}\right) / \mathrm{d} t$ on $P_{\text {RHESSI }}$ is found in these five events, and the slope is the average value ranging from $11.7 \%$ to $34.5 \%$ indicating this part of the kinetic energy of nonthermal electrons could be efficiently transformed into thermal plasma energy which could be traced by RHESSI SXRs. This is consistent with previous findings [13]. Therefore, the bulk of nonthermal energy input is lost during flaring; at least, this can not be determined from X-ray observations. Of course, these energies could heat the plasma at lower in the atmosphere to radiate in emissions at other wavelengths (i.e. $\mathrm{H} \alpha$ and UV) and to drive mass flows (i.e. chromosphere evaporation) during flaring.

In this paper, the thermal, nonthermal, radiation and conduction energies are estimated from RHESSI observations. Actually, accurate determination of these quantities from the present observations is not trivial. There are substantial uncertainties in the measurement. For example, the thermal energy is also sensitive to the filling factor, which cannot be observationally determined. The filling factor is assumed to be unity here. In general, there is significant evidence that the filling factor is less than one, perhaps in the range $0.001-$ 0.1 . Moreover, flare volume has great uncertainty because of the unknown scale length along the line of sight and the unknown source geometry. Actual flare scenarios show fine time-dependence structures within the loops and geometries. These uncertainties, and possible time dependence of volume and filling factor, introduce what is generally thought to be an order of magnitude uncertainty in the thermal energy [6]. In addition, the nonthermal energy flux depends sensitively on the value of the low-energy cutoff. The spectral fits in Figure 3 usually give an upper limit on the low-energy cut- off and, therefore, a lower limit on the energy flux in the nonthermal electrons. The radiation and conduction energies are computed using observational temperatures and emission measures, which already include contribution of the radiation, conduction, and other processes. The temperature scale length could be time-dependent during flare eruption. It is possible that $E_{\text {rad }}$ and $E_{\text {cond }}$ could represent lower limits. In such a case, the value of $\mu$ here is usually an upper limit to this ratio in actual physical scenarios.

This work was supported by the National Natural Science Foundation of China (10833007, 10973042, 11073058, 11073006, 40804034), National Basic Research Program of China (2011CB811400) and Key Laboratory of Dark Matter and Space Astronomy, Chinese Academy of Sciences (2010DP173032).

1 Neupert W M. Comparison of solar X-ray line emission with microwave emission during flares. Astrophys J, 1968, 153: 59-63

2 Hudson $\mathrm{H}$ S. Differential emission-measure variations and the "Neupert effect". Bull Am Astron Soc, 1991, 23: 1064-1066

3 Dennis B R, Zarro D M. The Neupert effect - What can it tell us about the impulsive and gradual phases of solar flares? Solar Phys, 1993, 146: 177-190

4 McTiernan J M, Fisher G H, Li P. The solar flare soft X-ray differential emission measure and the Neupert effect at different temperatures. Astrophys J, 1999, 514: 472-483

5 Veronig A, Vršnak B, Dennis B R, et al. Investigation of the Neupert effect in solar flares. I. Statistical properties and the evaporation model. Astron Astrophys, 2002, 392: 699-712

6 Veronig A M, Brown J C, Dennis B R, et al. Physics of the Neupert effect: Estimates of the effects of source energy, mass transport, and geometry using RHESSI and GOES data. Astrophys J, 2005, 621: 482497

7 Ning Z J. RHESSI observations of the Neupert effect in three solar flares. Solar Phys, 2008, 248: 99-111

8 Cargill P J, Mariska J T, Antiochos S K. Cooling of solar flares plasmas. 1. Theoretical considerations. Astrophys J, 1995, 439: 1034-1043

9 Ning Z J. Different behaviors between microwave and hard X-ray spectral hardness in two solar flares. Astrophys J Lett, 2007, 671: 197-200

10 Warmuth A, Holman G D, Dennis B R, et al. Rapid changes of electron acceleration characteristics at the end of the impulsive phase of an X-class solar flare. Astrophys J, 2009, 699: 917-922

11 Gan W Q, Fang C. A hydrodynamic model of the gradual phase of the solar flare loop. Astrophys J, 1990, 358: 328-337

12 Sui L, Holman G D, Dennis B R. Evidence for magnetic reconnection in three homologous solar flares observed by RHESSI. Astrophys J, 2004, 612: 546-556

13 Saint-Hilaire P, Benz A O. Thermal and non-thermal energies of solar flares. Astron Astrophys, 2005, 435: 743-752

14 Ning Z J. RHESSI microflares with quiet microwave emission. Astrophys J, 2008, 686: 674-685

15 Ning Z J. The investigation of the Neupert effect in two solar flares. Sci China Ser G: Phys Mech Astron, 2009, 52: 1686-1690

16 Ning Z J, Cao W. Investigation of the Neupert effect in the various intervals of solar flares. Solar Phys, 2010, 264: 329-344

17 Liu L B, Wan W X, Chen Y D, et al. Solar activity effects of the ionosphere: A brief review. Chin Sci Bull, 2011, 56: 1202-1211

18 Li R, Wang H N, Cui T M, et al. Solar flare forecasting using learning vector quantity and unsupervised clustering techniques. Sci China Phys Mech Astron, 2011, 54: 1546-1552 
19 Lin R P, Dennis B, Hurford G, et al. The Reuven Ramaty High-Energy Solar Spectroscopic Imager (RHESSI). Solar Phys, 2002, 210: $3-$ 32

20 Hurford G J, Schmahl E J, Schwartz R A, et al. The RHESSI imaging concept. Solar Phys, 2002, 210: 61-86

21 Smith D M, Lin R P, Turin P, et al. The RHESSI spectrometer. Solar Phys, 2002, 210: 33-60

22 Oberoi D, Evarts E R, Rogers A E E. High temporal and spectral resolution interferometric observations of unusual solar radio bursts. Solar Phys, 2009, 260: 389-400

23 Wang H, Liu C, Jing J, et al. Successive flaring during the 2005 September 13 eruption. Astrophys J, 2007, 671: 973-977

24 Liu C, Lee J, Karlický M, et al. Successive solar flares and coronal mass ejections on 2005 September 13 from NOAA AR 10808. Astrophys J, 2009, 703: 757-768

25 Nagashima K, Isobe H, Yokoyama T, et al. Triggering mechanism for the filament eruption on 2005 September 13 in NOAA active region 10808. Astrophys J, 2007, 668: 533-545

$26 \mathrm{Li} \mathrm{H}$, Schmieder B, Song M T, et al. Interaction of magnetic field systems leading to an X1.7 flare due to large-scale flux tube emergence. Astron Astrophys, 2007, 475: 1081-1091

27 White S M, Thomas R J, Schwartz R A. Updated expressions for de- termining temperatures and emission measures from GOES soft X-ray measurements. Solar Phys, 2005, 227: 231-248

28 McTiernan J M. RHESSI/GOES observations of the nonflaring Sun from 2002 to 2006. Astrophys J, 2009, 697: 94-99

29 Sui L, Holman G D, Dennis B R. Determination of low-energy cutoffs and total energy of nonthermal electrons in a solar flare on 2002 April 15. Astrophys J, 2005, 626: 1102-1109

30 Hannah I G, Christe S, Krucker S, et al. RHESSI microflare statistics. II. X-ray imaging, spectroscopy, and energy distributions. Astrophys J, 2008, 677: 704-718

31 Fisher G H, Hawley S L. An equation for the evolution of solar and stellar flare loops. Astrophys J, 1990, 357: 243-258

32 Ji H S, Song M T, Hu F M. A unified model for the Sun's lower and upper transition regions. Astrophys J, 1996, 464: 1012-1016

33 Jiang Y W, Liu S, Liu W, et al. Evolution of the loop-top source of solar flares: Heating and cooling processes. Astrophys J, 2006, 638: 1140-1153

34 Aschwanden M J. RHESSI timing studies: Multithermal delays. Astrophys J, 2007, 661: 1242-1259

35 Culhane J L, Phillips A T, Inda-Koide M, et al. YOHKOH observations of the creation of high-temperature plasma in the flare of 16 December 1991. Solar Phys, 1994, 153: 307-336

Open Access This article is distributed under the terms of the Creative Commons Attribution License which permits any use, distribution, and reproduction in any medium, provided the original author(s) and source are credited. 